\title{
A Review of New Technologies: AR, VR, MR
}

\author{
Maithili Shah \\ DJ Sangvi College of Engineering \\ Vile Parle West \\ Mumbai- 400056, India
}

\author{
Parth Mehta \\ DJ Sangvi College of Engineering \\ Vile Parle West \\ Mumbai- 400056, India
}

\author{
Neha Katre \\ DJ Sangvi College of Engineering \\ Vile Parle West \\ Mumbai- 400056, India
}

\begin{abstract}
Computer Graphics basically means creating and manipulating images to produce interactive images, animations, etc. Use of computer graphics led to development of Augmented Reality, Virtual Reality and Mixed Reality. Applications of these realities is increasing day by day and is likely to become future of human race. This paper reviews all these realities, describing the working, various devices and the applications of them. It also finds points of distinctions among them.
\end{abstract}

\section{Keywords \\ AR, VR, MR}

\section{INTRODUCTION}

The term "computer graphics" refers to anything involved in the creation or manipulation of images on computer, including animated images [1]. It can be used for animation, design, visualization, special effects, games in computer, interaction purposes. Use of computer graphics has increased day by day. New techniques were developed to convert computer graphics into interactive computer graphics. It led to the development of new emerging technologies like augmented reality and virtual reality. Computer graphics leads the user to a new reality to be a part of a world and experience things which seem practically impossible in real life and in a completely different environment or dimension. Moreover, it creates a world free of limitations and constraints which can be manipulated as per the user's need. It can be called the dimension of imagination. A revolution in design interface of computers has changed the mindsets about computers. Through AR user can see the real world with virtual objects superimposed upon it. Rather than typing on a keyboard and watching a television monitor, Augmented Reality lets people use familiar, everyday objects in ordinary ways. Along with Augmented reality, concepts of a different world, a virtual world also came into existence. In the early 19902s due to the increasing development in VR, it started becoming very famous. Virtual Reality (VR) is a popular name which uses a special human-computer interface in which user experiences an interactive, computer-mediated synthetic environment. The simulated objects can be interacted as real life objects. Virtual shared environment allows the users to see one another and interact with one another as they were in same environment. Reality is something that is present in real or constitutes a real, something that is present independently. Virtual is defined as something almost equal to real but not real. As advancements in field of virtual reality increased, new ideas and experiments to develop a combination of AR and VR also started developing. This led to origin of Mixed Reality. It is an enhanced reality that works seamlessly between Virtual Reality (VR) and Augmented Reality (AR). The principle distinction amongst $\mathrm{VR}$ and $\mathrm{MR}$ is that $\mathrm{MR}$ tries to consolidate reality with PC created imagery to such an extent, to the point that the two turn out to be practically in differentiable. The capability to change between a mode that is totally immersive and other that superimposes to the customer's surroundings is the thing that makes MR not the same AR. Mixed Reality is also known as hybrid reality which is a combination of virtual world and real world where computer and physical exist together and interact in real time to produce new visual environments. MR is an exceedingly interdisciplinary field connecting with PC vision, PC illustrations, UIs, human elements, wearable processing, portable registering, information representation, the plan of showcases and sensors.

Having looked at the basic concepts of AR, VR and MR; the rest of the paper is organized as follows, Section 2 discusses the working of augmented reality, its applications and the devices used. Section 3 throws light on the working of virtual reality, its applications and devices. Discussion on mixed reality is given in section 4 . Section 5 compares the three realities and section 6 concludes the paper.

\section{AUGMENTED REALITY}

The objective of an AR framework is to improve the client's impression of and cooperation with this present reality by supplementing this present reality with $3 \mathrm{D}$ virtual articles that seem to exist together in an indistinguishable space from this present reality. AR comprises of taking after three attributes:

1) Joins genuine and virtual

2) Interactive progressively

3) Registered in 3-D [2].

\subsection{Working}

Creating an Augmented Reality experience is a multi-stage process, from the development of the 3D models to the technology that powers the image tracking and depth sensing. Each step is an intuitive process. 3D artists use several software programs like Sketch Up, Cinema 4D, Blender and many more to create 3D models. The process starts with a rough sketch after which it goes through various series of approval that helps in refining the idea and thus leads to complete image. After the image is accepted, the modelling begins. Next step is to apply the model's skin, which is called the texture map. Texture map gives realism to the model and can be styled to fit the needs of the project. After the completion of the 3D model, it goes through the rendering process for a complete AR experience [3].

\subsection{Applications}

\subsubsection{Medical}

Augmented Reality provides hidden information to the surgeon like rate of the heartbeat, state of the person's organ, blood pressure etc. Augmented Reality can be useful by allowing a doctor to look inside the patient by combining one source of images like an X-ray with another one such as 
video. It can also enhance viewing of a fetus inside a mother's womb [4].

\subsubsection{Military}

Augmented reality can be used to display the real battlefield scene. Arcane developed an animated terrain that could be used for military intervention with the help of augmented reality. For example, a soldier, could use special type of indicators to mark various kinds of objects, to warn them of potential dangers [4].

\subsubsection{Marketing}

Automotive industry had firstly used AR for various marketing purposes. Various companies had printed special type of flyers that were recognized by webcams. They would create a 3D model that was then displayed on the screen. Similar kind of approach was used for marketing purposes in various fields. A new marketing strategy was developed using the shoes where the user wears a special pair of socks and then when the user walks in front of a camera he is able to see on the screen his image sporting the pair of shoes he wishes to try or buy [4].

\subsubsection{Navigation}

Augmented reality can be used for various route gadgets. Data can be shown on the windshield to indicate meter, terrain, destination directions, weather, traffic and road conditions and alerts to potential hazards in their path. It can also allow bridge watch-standers to continuously monitor important information like a ship's heading and speed while moving through the bridge or performing any other tasks [4].

\subsubsection{Entertainment}

Augmented reality has its application in the field of entertainment it can be used in the field of games, sports like (swimming pools, football fields, race tracks and other sports environment), virtual ads, product placements and many more. For example, Fox-Trax system used for ice hockey, various AR games like Quake, Game City and many more [4].

\subsubsection{Education and Training}

Embedded markers can be used in educational materials which when scanned by an AR device produces information in multimedia format which helps the student to study various subjects [4].

\subsubsection{Office}

Collaboration in office spaces is another zone where AR might be valuable by giving joint effort among different colleagues in a work compel by means of meetings with the assistance of genuine and virtual members. Its undertakings incorporate conceptualizing and exchange gatherings using basic representation with the assistance of intelligent advanced whiteboards, touch screen table and shared outline spaces [4].

\subsection{Devices}

\subsubsection{Head Mounted}

HMD's are usually paired with headset like helmet or harness. It places the image of the virtual objects as well as the physical world above the user's view. Modern HMDs consists of sensors having six degrees of freedom which allow it to align the virtual information to the physical world and can accordingly adjust as per the user's head movements. It provides the users with mobile, immersive, collaborative experiences [4].

\subsubsection{Eyeglasses}

It includes an eye wear that enrolls cameras to capture reality and it re-displays the reality with augmented view with the help of eye pieces and devices which reflect the projected image [4].

\subsubsection{Contact lenses}

These are being developed. They are bionic contact focal points that may contain the components for show installed into the focal point, including LEDs, a radio wire for remote correspondence, coordinated hardware [4].

\subsubsection{Virtual retinal display}

It is a personal display device which is under development at the Human Interface Technology Laboratory in the University of Washington. With the help of this technology, the display can be scanned directly onto the retina of the watcher's eye. The watcher sees it in the form of a conventional display floating in space in front of him [4].

\subsubsection{Eye Tap}

Alternatively termed as, Generation-2 Glass which seizures the beam of light and substitutes each ray of light for synthetic computer-controlled light. It causes the eye to function both as a camera as well as a display [4].

\subsubsection{Handheld}

Handheld is a small display that can fit in the user's hand. Initially handheld AR employed fiduciary markers, and later GPS units and MEMS sensors such as digital compasses and six degrees of freedom accelerometer gyroscope. AR handheld has two principle focal points that it is the convenient way of handheld gadgets and pervasive nature of camera telephones. The burdens are the physical limitations of the client holding the handheld gadget dependably before them [4].

\subsubsection{Spatial}

Spatial enlarges certifiable articles and scenes without the use of HMDs, screens or hand-held devices. It makes utilization of advanced projectors to demonstrate the graphical information onto physical articles. Cases of SAR are portable projectors, shader lights, virtual tables, keen projectors. A graphical discernment and idle haptic sensation for the end customers are both maintained by SAR [4].

\subsubsection{Tracking}

There are variety of tracking technologies like accelerometers, digital cameras, GPS, gyroscopes and wireless sensors. They offer diverse levels of precision and accuracy [4].

Having looked at the working, applications and various devices of AR, now let's look at the various concepts of VR.

\section{VIRTUAL REALITY}

Reality can be defined as something which is present in real or constitutes a real, something that is present independently. Virtual is defined as something almost equal to real but not real.

\subsection{Working}

Eyes are a sure width separated, which implies when somebody takes a gander at a protest, each eye sees a somewhat unmistakable point of view of that question. Their mind then procedures these two pictures to frame a 3D picture. VR gadgets work similarly. The initial segment of the procedure is recreating both the left and right eyes' points of view with two one next to the other pictures. This is known as 
'stereoscopic imaging'. The second some portion of the procedure is to utilize an OLED show of high determination and sufficiently huge to make an immersive field of vision. At the point when these are joined a radical new world in framed before the eyes. The immersive picture is the gadget's capacity to track the head development with no slack or inertness as the client checks out the virtual world. This requires a few sensors, including gyro meters, accelerometers and that's just the beginning. For Virtual Reality headsets, they screen the correct position of client's head over the 'six degrees of flexibility' i.e. two headings of pitch, yaw and roll [5].

\subsection{Applications}

In the last few years, people have shown a lot of interest in VR. It is considered as a new prototype that offers many advantages in various areas of application. Human-computer interaction has become easy and powerful due to VR.

\subsubsection{Modeling, designing and planning}

Virtual Reality offers users to view virtual objects in real time and in real space in a virtual environment. For instance, the models developed at the Fraunhofer Institute Virtual Design, Virtual Kitchen - tools for interior designers who can visualize their sketches. They can change colors, textures and positions of objects, observing them instantaneously to see how the whole surrounding would look like [6].

\subsubsection{Training and education}

Use of VR is increased in civil industries also as they are safe and provide lower operating cost than the real flight training. Therefore, astronauts were trained using virtual reality as it is more efficient and they can also perform hazardous tasks in space without any fear. Another application is medicine where they allow study and training of performing surgeries of various parts of body. Another example is Virtual classes; where student present at any place can study with virtual teachers [6].

\subsubsection{Telepresence and Teleoperation}

Telepresence technology lets users to do medical operations in a faraway widely different condition with the help of VR user connections. The Nan manipulator undertaking shows a different quality of telepresence in faraway widely different operating conditions. This system that uses a HMD and forcefeedback taking care of expertly lets a man of science to see a microscope view, have a feeling of and make use of, do something with the top of the representative of condition [6].

\subsubsection{Cooperative working}

Remote users can easily collaborate with each other using network based virtual environment. The higher bandwidth of information sharing can be used for cooperative working. Some practical applications are a collaborative CO-CAD desktop system that allows a group of engineers to work together within a shared virtual workspace [6].

\subsubsection{Entertainment}

Field of entertainment is also a large user or application of virtual reality due to the constantly increasing power of hardware and decreasing prices [6].

\subsection{Devices}

\subsubsection{Position and orientation tracking devices}

\subsubsection{Magnetic trackers}

Magnetic trackers generally comprise of following parts: a control station unit, a stationary part (source like the emitters) and movable parts (receivers generally called sensors). Emitter and receiver both use three antennae which are 90 degrees to each other and are assembled in analogous manner. The receiver's antenna picks up the magnetic fields generated by the current provided to emitter antenna. The receiver sends its measurements (nine values) to the control unit that calculates position and orientation of the given sensor. They are very useful as they are small and light in weight and thus easy to handle [6].

\subsubsection{Acoustic (ultrasonic) trackers}

Ultrasonic waves (above $20 \mathrm{~Hz}$ ) are used by Acoustic trackers for determining the location and orientation of object in space. Acoustic trackers are of typically of two types-they either use phase-coherent (PC) or time-of-flight (TOF) measurements to evaluate separation of pair of points. TOF trackers are also used to analyze the flight time of small ultrasonic pulses between source and sensor. Computerized trackers compare the phases of reference and received signals. The phase difference is 360 degrees which is proportionate to the separation of one wavelength. [6].

\subsubsection{Eye tracking}

\subsubsection{Limbus tracking}

The sharp limit between sclera (limbus) and iris can be effortlessly distinguished. The infrared light emitting detectors and phototransistors are fixed on the client's eyeglasses to screen the infrared spots reflections from the iris and limbus to decide the look heading [6].

\subsubsection{Image tracking}

In this procedure, the look course is controlled by the camcorder and picture preparing strategies. This innovation offers great precision around $1^{\circ}$. It is currently used by ISCAN, Applied Science Labs 4000 SU-HMO [6].

\subsubsection{Electro-oculography:}

This strategy utilizes the terminals which are put other than the eyes to gauge the standing potential amongst cornea and retina. Ordinarily, the recorded possibilities are little which lies between $15 \mu V$ to $200 \mu V$ [6].

\subsubsection{Corneal reflection:}

This method utilizes photograph transistors to examine an impression of collimated light emission from the curved cornea surface. This approach offers generally better exactness of 0.5 degree to 1 degree, yet requires more intricate adjustment, covers moderately little eye development region [6].

\subsubsection{D input devices:}

Gloves is a 3D input apparatuses which is used to find the various angles of fingers. They use various optical fiber sensors like VPL Data Glove, foil-strain technology gloves like Vertex Cyber Glove or resistive sensors like Mattel Power Glove to measure the finger flexion. The use of gloves lets the user a better and fuller effect than the 3D instrument for pointing (used for computers), because it provides proper translation of the hand gestures and various actions. They are also furnished with a tracker which is attached to the client's wrist to measure its position and adjustment [6].

Having looked at the concepts of Virtual Reality, now let's see the working, application and devices of Mixed Reality.

\section{MIXED REALITY}

Mixed reality is a mixture of Virtual reality and augmented reality. It is also referred as hybrid reality. It merges real and 
virtual worlds to produce new visualizations and environments where digital and physical objects interact in real-time.

\subsection{Working}

Mixed Reality devices work by creating a 3D map of user's surroundings and scanning the physical environment so that the device knows how and where to put the computerized content into that space realistically while allowing the client to collaborate with it using motions. MR experiences invite computerized content into user's real-time environment, allowing the user to collaborate with them. The holograms can behave like real world objects and interact with surroundings around them due to the use of transparent lenses, understanding of physical environments and sound. For example, think of the aliens crashing through the roofs and firing missiles, weapons at the user. It is not only breathe taking but also a thrilling experience [7].

\subsection{Applications}

\subsubsection{Industries}

In 1993, Steve Feiner and his crew at Columbia University were the initial ones to develop a MR system that would guide the users in basic maintenance and repairing operations. Rhee, Lee presented MR system that was distributed and collaboration oriented. Their framework incorporates cell phones and in addition PC terminals, associated with a server and a metaphysics based setting acknowledgment framework to render the data in the configuration fitting to the customer and the circumstance. Another application called Magic book built 3-D models of objects on the pages of the book. Different angles of the models can be seen by moving the book and observing from different angles [8].

\subsubsection{Medicine:}

MR is useful in medical field for directly visualizing medical images (for e.g., CT scans, MRI) of various body parts of the patient to guide the surgeon to operate the patient. Monitors in the operating room are used to display these medical images. A survey has shown that the accuracy can be increased by using such technologies with respect to traditional methods [8].

\subsubsection{Collaboration:}

It is another zone of application for mixed reality. Here for example, a remote expert gives guidance to an operator on field. The operator makes use of mobile mixed reality to capture the scenario around him and forward it to the expert which analyses it and guides the operator accordingly through visual display of the operator's mixed reality system or the audio channel. For medical uses, Welch et al had suggested a system which visualizes the patient's body captured by multiple cameras and then displayed on a high resolution display or PDA of an expert at his place [8].

\subsubsection{Navigation:}

For navigation, mixed reality systems can be used to provide assistance. In this situation, operators can perceive virtual symbols anchored to the real world. It is quite like a compass where the signs show the proper direction without getting affected by the orientation of the gadget. It could be utilized to guide guests inside the house or buildings or for travelers in a new city [8].

\subsubsection{Entertainment:}

Several exciting applications were suggested, in which handlers must interact with synthetic characters or gadgets which appear in their real environment. Mixed reality can be used in games which help to increase the competition as well as collaboration among the players. For example, MR version of the Quake video game, the Human Pac man game, Moon Lander game, etc. [8].

\subsection{Devices}

\subsubsection{HoloLens}

The HoloLens creates an artificial sensory experience like something out of a sci-fi film, albeit a basic one. The new offering from Microsoft projects holograms into real time environments that can only be observed if the user wears the all-in-one device. There is no need for headphones in HoloLens as it is fully equipped with everything that is required for a semi-immersive experience. It consists of environment and depth sensors that will allow it to process real time information [9].

Now after going through all the three types of Realities, namely AR, VR and MR, the next section compares AR, VR and MR to find out various points of distinction between them.

\section{COMPARISON 5.1 Virtual Reality}

\subsubsection{Features}

To enhance the experience and make virtual reality exciting devices are often used to block out the real world. It's a totally immersive experience allowing user to interact with a completely different world [10].

\subsubsection{Platform}

It has already been used to help with training medical staff and military as well as in gaming to give an experience on a completely different level. More recently virtual reality has stepped up to the next level offering users a more realistic experience to lose themselves in different world [10].

\subsubsection{Headset}

By using a headset people can walk around their own surroundings yet feel as though they are in a completely different place to their reality. They can interact with other users in games and digital characters without the outside world intruding [10].

\subsubsection{Prospect}

Aeronautical and scenario simulation has already been able to utilize virtual reality as a tool for training without having to be in a real-life situation. In the gaming world, it takes people out of their real world and gives them a totally unique experience [10].

\subsection{Augmented Reality}

\subsubsection{Features}

AR gives user an indirect view of a real-life scenario with the help of digital technology. For example, there is a vacant room yet the user can see how it would appear in various colors even without purchasing them with the help of digital technology. The experience can be made more realistic by using sound and other sensory inputs [10].

\subsubsection{Platform}

Even though the digital and other augmented sections of the augmented reality are superimposed in real life, they are not actually part of it. It can be used to interact with the real world to help make recommendations of improvement such as recipe 
ideas based on real ingredients laid out before the camera [10].

\subsubsection{Headset}

A headset can improve the experience further by adding characters in a game that can speak, give suggestions, thus immersing user in part to give him the feel of realism, thus describing it as a real life overlay rather than a thorough digital and sensory experience that takes user away from it [10].

\subsubsection{Prospect}

Augmented Reality is being used in day to day real life situations like training. It is also being used in gaming and more recently with mobile technology using GPS signals to add more realism and accuracy [10].

\subsection{Mixed Reality}

\subsubsection{Features}

MR brings the digital world and real world together to bring a new experience. Rather than just being added to the real world the experiences instead are interactive to a point where they can be used in many applications. Characters and scenarios can be mixed with real world surroundings

[10].

\subsubsection{Platform}

As digital scenarios take place in real world surroundings, it gives user a better sense of realism. This feature can be used in many places like in training and in gaming technology [10].

\subsubsection{Headset}

Using a headset allows for a better sense of realism. But since it is a mixed reality not all scenarios would be suitable for the headset to interact with others who would be physically present [10].

\subsubsection{Prospect}

Holograms have been used more recently to make the user feel as though an object or character is present. Artificial content is being improved all the time, which shows that the future of mixed reality is not far away. It's the closest that technology has been so far to creating a novel experience [10].

\section{CONCLUSION}

In this paper, we have reviewed the working, applications and devices of Augmented Reality, Virtual Reality and Mixed
Reality. This paper also analyzes the various parameters of distinction between them. MR and AR, while labeled differently, are virtually very similar in practical life and applications. They would permit the users to live through an actual world while it overlays a computerized interface in actual-time. They make the world around the user, a canvas in which the user can play, interact and learn. Collectively, all three realities are very useful and have great applications presently and in time to come.

\section{ACKNOWLEDGEMENT}

We would like to acknowledge Mrs. Neha Katre for her guidance and efforts to guide us throughout the writing of the paper.

\section{REFERENCES}

[1] Introduction: http://math.hws.edu/graphicsbook/c1/index. html

[2] Ronald Azuma, Yohan Baillot, Reinhold Behringer, Steven Feiner, Simon Julier, Blair MacIntyre, "Recent Advances in Augmented Reality", Computer and Graphics, November 2001.

[3] E-book on Augment's Essential Guide to Augmented Reality.

[4] Augmented Reality-Crystal Links.

[5] Working: VR,'https:// blog.vodafone.co.uk/2016/02/18/ tech-trivia how- virtual-reality-works/"

[6] Tomasz Mazuryk and Michael Gervautz, "Virtual Reality History, Applications, Technology and Future", Institute of Computer Graphics Vienna University of Technology, Austria. http://www.cg.tuwien.ac.at/.

[7] Working:MR,'https://thenextweb.com/insider/2017/01/0 7/mixed reality-will-be-most-important-tech-of2017/\#.tnw_6a31CM81".

[8] Enrico Costanza, Andreas Kunz,and Morten Field, "Mixed Reality “A Survey”, 2009.

[9] Hololens,http://www.information-age.com/mixed-reality.

[10] Comparison between AR, VR and MR, https://filmora.wondershare.Com/virtualreality/difference -between-vr-ar-mr. 